Goudie A:

\section{The human impact on the natural environment}

2005. 6th edition; Blackwell Science Publishing; 357 pp., 240 figs., 99 tables; Paperback USD 39.95; GBP 24.99; ISBN 1-4051-2704-X

Given the multitude and partly magnitude of human impacts on the natural environment, it may appear impossible to write a book with such a title. On the other hand, it is a demanding necessity to provide exactly such information to a wide group of readers - from students to decision makers on many levels.

Andrew Goudie has taken on that challenge already in 1981 with the first edition of this volume. Now the sixth edition is a clear reflection of how necessary this is. This new edition has been largely updated and enlarged with new four chapters primarily to address the topics of global climate change and its layout has been changed to improve readability and practicality.

The book is organized in two parts with a total of 13 chapters. Part I, entitled "The Past and Present", delivers an introduction (1) with a review of the human development; (2) the human impact on vegetation, (3) human influence on animals, (4) the human impact on soil, (5) on the waters, (6) human activity in geomorphology, and (7) the human impact on climate and the atmosphere. Part II, "The Future", discusses following an (8) introduction, (9) coastal environments, (10) hydrologic impacts, (11) the cryosphere, (12) drylands, and sums up with a comprehensive conclusion that poses several very critical questions on the sometimes open questions of a predisposition of natural environments by long-term usage in cultural development, the proliferation of impacts and their reversibility, and the general question concerning a differentiation between human impact and natural variability and forces that are sometimes conveniently overlooked. The book closes with a short glossary, a substantial list of references, and a helpful subject index.

The book is filled with new information and intriguing examples that Goudie has selected to illustrate in each of his well-organized chapters. One may not always be in full agreement with the author, but the reader will certainly find plenty of food for thought and is often assisted by the so-called "points for review" at the end of each chapter that provide questions related to the understanding of the previous topic. As with the previous editions of this book, this book serves to improve a more holistic view of the Earth system and its feedback mechanisms in the low PT sphere of its crustal surface for both students and teachers. For the reader of Environmental Geology, there will be plenty of useful information that may help in interpreting own data and results from a broader viewpoint. Despite its volume, this book is an easy-to-read primer for decision makers on the topic.

Severin KP:

\section{Energy dispersive spectrometry of common rock forming minerals}

2004. Kluwer Academic Publishers; 225 pp., 191 illus.; Hardcover with spiral binding; USD 169.00; EUR 139.05; GBP 79.00; ISBN 1-40202840-7

Everyone using scanning electron microscopy with EDX and anyone who is not the expert (knowing the spectra by heart) will thoroughly appreciate this novel book with flashcard-like spectra for $182 \mathrm{~min}$ erals. As the author states, he did not aim for a complete assembly of all or even most minerals, but focused on those that are commonly referred to as the more common major rock forming minerals, as described in textbooks, such as Deer, Howie and Zussmann's Introduction to Rock-forming Minerals.

The first chapter provides a 13page overview on the principles of energy dispersive spectrometry with the how's and why's of the application with scanning electron microscopy. Thus, it does not aim at much more but gives a little background to the technique and its options. The second chapter deals with sample preparation, and spectra acquisition and interpretation. This chapter is a ten-page primer into the basics that any user should be aware of. The third chapter offers an 11-page key to quickly find the correct or most likely mineral to the obtained spectrum. It basically works like many plant or rock determination books for beginners with clearly given questions and the yes/no answers that lead the user to the next question until he has found the right or most probable result, divided into silicates and non-silicates. Chapter 4 is the real core of this handy volume with mineral spectra from Olivine to Sylvite. Most spectra were obtained using a Be-window on natural mineral phases using a $15 \mathrm{keV}$ accelerating voltage. For different examples of a solid-solution series, at least examples for the end members are given. The book closes with a few references, and an index to spectra, minerals and mineral groups to ease finding a specific spectrum.

It may be clear from the given description that this useful work is a true hands-on practical guide particularly to anyone (student or professional) working occasionally or with limited experience with SEMEDX. For those it will quickly become an almost indispensable reference that helps to identify minerals with more security and thus take less time from the supervisor. It does not, however, replace the experienced spectroscopist, nor does the author claim that his work does so. It is a help, and as such, most welcome and will certainly find many friends. 


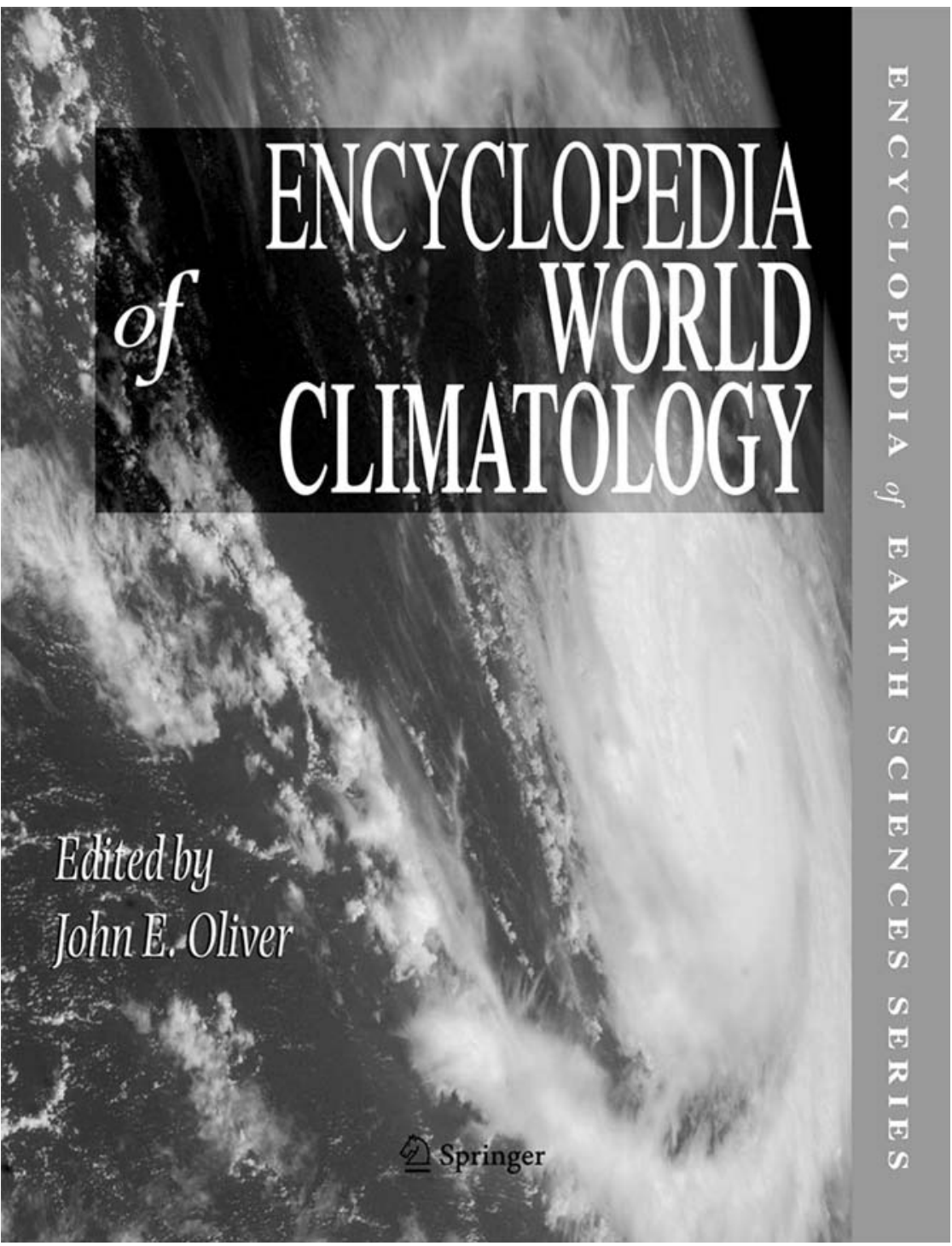

Oliver JE (ed):

\section{Encyclopedia of world climatology}

2005. Springer; 854 pp., 420 illus.; Hardcover (without sales tax) USD 499.00; EUR 399.00; GBP276.00; ISBN 1-4020-3264-1

In 1999 Kluwer Academic Publishers produced the excellent Encyclopaedia of Geochemistry - a volume of an ambitious series. This brand-new volume, Encyclopedia of World Climatology, is another addition to the series and is executed in a similar fashion and with the same high quality standards. Since it becomes ever more challenging to maintain an overview of the large individual fields of science, the decision of the editors to provide the community with this series cannot be overapplauded. The enormous work behind the volume hopefully will be honored by regular updates.

For this volume, the volume editor, John E. Oliver, has brought together 163 authors to contribute to this remarkable volume. From "Acid Rain" via the "Madden-Julian Oscillation" to "Zonal Index", 231 contributions fill this volume with life. A 19-page index of the authors cited (to be able to retrieve specific literature within this fat volume) and a 12-page subject index complete the encyclopedia. Each contribution contains a bibliography and crossreferences to other parts of this volume. It would be unfair to spotlight individual contributions as examples; an encyclopedia needs to be assessed in its breadth.

While all tables are very well set, many figures based on scans are less than optimal. Quite a few references relate to internet-based information - a source that while increasingly common, still leaves one with an uneasy feeling (since it happens ever so often) that some fine information may simply no longer be available at a given site - even if the site is being maintained by a state agency or high-ranking scientific institution. Apart from this minor criticism, this encyclopedia is a wonderful and most valuable information source. It is a "must-have" for every library and should be within hands reach of those working in climate research.

Jörg Matchullat

Interdisciplinary Environmental

Research Center

TU Bergakademie

Freiberg

Germany

\section{Geo-surfing: Internet sites for environmental geology}

\section{http://www.elementsmagazine.org}

This is the website of the bimonthly magazine, Elements. This international magazine of mineralogy, geochemistry, and petrology was launched in January 2005 by the Mineralogical Society of America, the Mineralogical Society of Great Britain and Ireland, the Mineralogical Association of Canada, the Geochemical Society, the Clay Minerals Society, the European Association for Geochemistry, and 


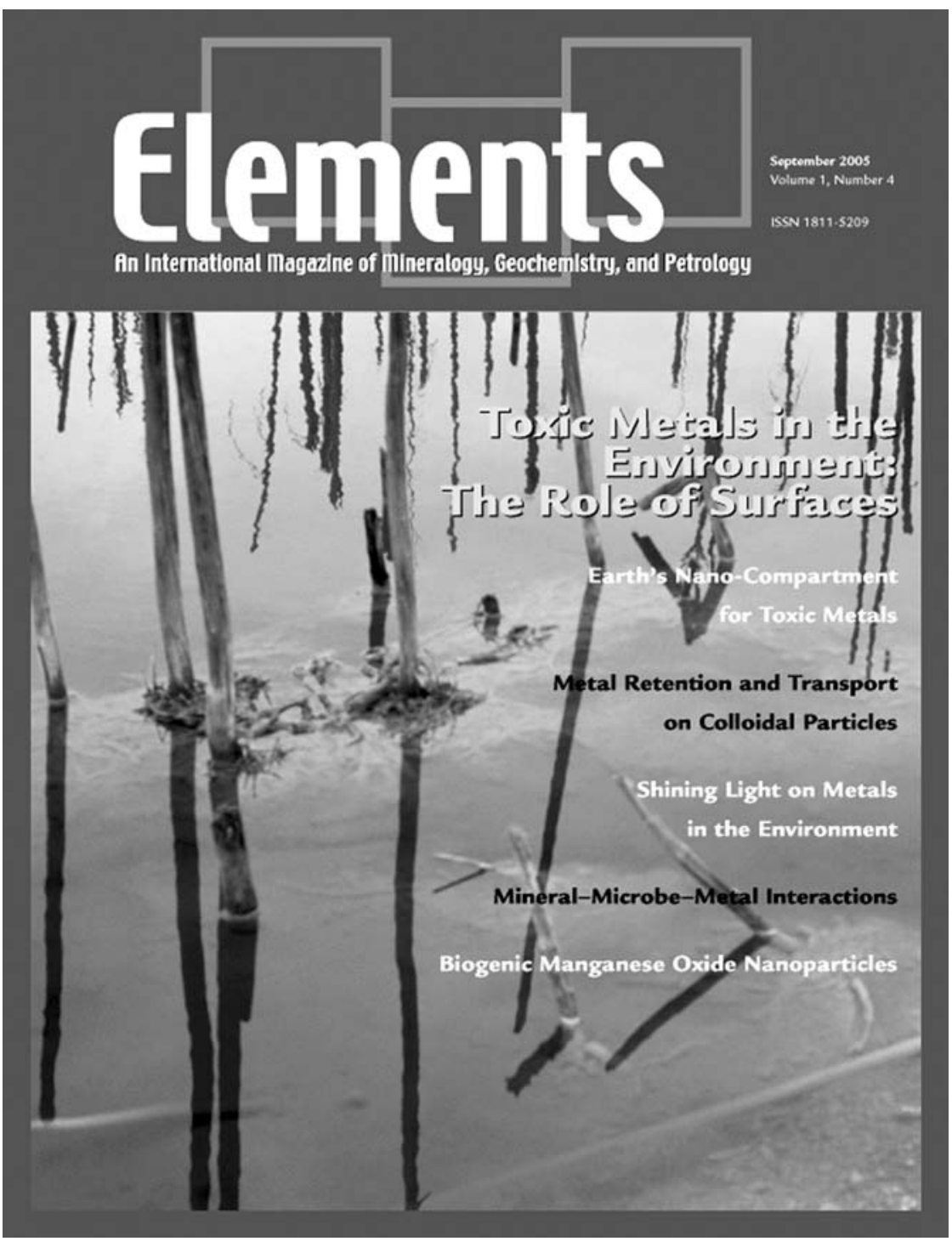

the International Association of Geochemistry. The Société Française de Minéralogie et de Cristallographie will join this international group in 2006.

Each issue explores a theme of broad and current interests in the mineral and geochemical sciences. The magazine invites peer-reviewed papers for each thematic collection of articles. Themes of interest can be proposed to the editors and the members of the advisory board who review each proposal submitted.
The 2005 issues focused on themes such as: Rocks, minerals, and the geochemical origin of life; Toxic metals in the environment the role of surfaces; Large igneous provinces and environmental changes. Themes for the upcoming issues of 2006 have been announced; these are:

- User research facilities in the Earth sciences

- Arsenic

- Water on Mars

- Early Earth

- Glasses and melts: linking geochemistry and materials sciences

- Environmental aspects of the nuclear fuel cycle

The editors of Elements also regularly present a calendar of events, announce short courses, highlight groundbreaking papers and awards, and report on conferences, policy news, and the news of the Societies. Although this is not an e-journal, sample issues may be downloaded at no cost from the website, http:// www.elementsmagazine.org.

All members of the participating societies receive this magazine free. An annual fee of US \$125 is requested from library and corporate subscribers.

Anne Marie de Grosbois Assistant Editor 